\title{
Software-in-the-loop testing of a distributed optimal scheduling strategy for microgrids' aggregators
}

\author{
Fabio Bonassi, Alessio La Bella, \\ Lorenzo Fagiano, Riccardo Scattolini \\ Dipartimento di Elettronica, Informatica e Bioingegneria \\ Politecnico di Milano \\ Milano, Italy \\ name.surname@polimi.it
}

\author{
Donato Zarrilli, Pablo Almaleck, \\ Pietro Serra \\ Power Grids Division \\ ABB S.p.a. \\ Genova, Italy \\ name.surname@it.abb.com
}

\begin{abstract}
Recent regulations have explicitly endorsed the provision of ancillary services by Microgrids (MGs). However, the associated technical requirements (e.g. minimum power reserve) still represent an impeding factor for most MGs given their reduced capability. For this reason, approaches to pool MGs into Aggregators, allowing to jointly coordinate MGs to fulfill such requirements, have been proposed but their practical feasibility has been not proved. Therefore, the goal of this paper is to test a previously proposed distributed day-ahead scheduling algorithm on a realistic benchmark with ABB e-mesh ${ }^{\mathrm{TM}}$ EMS, an industrialgrade MG energy management system. The results show that the approach can deal systematically with different MG units and controllers, maintaining its scalable and optimal performances.

Index Terms-Microgrids, Distributed scheduling, Testing
\end{abstract}

\section{INTRODUCTION}

$\mathbf{I}$ $\mathrm{N}$ recent years, the surging necessity to reduce carbon dioxide emissions in favor of a sustainable energy mix based on Renewable Energy Sources (RESs), boosted the adoption and integration of Microgrids (MGs) into the Power System. MGs are small-scale grids - equipped with a MG Energy Management System (EMS), local loads, and Dispatchable Energy Sources (DERs) such as generators and batteries which can either operate in islanded mode, i.e. disconnected from the main grid, or in grid-connected mode. When connected to the main grid, MGs are entitled of performing the so-called Day-Ahead Scheduling (DAS), i.e. scheduling the internal dispatchable units so as to maximize the profit, based on the expected energy prices and on loads' and RESs' dayahead forecasts [1]. In the near future, MGs are also expected to support the power system operations by providing valuable ancillary services, such as offering power reserves, i.e. margins for the increase or decrease of MG's output power, that can be requested by the System Operator (SO) for balancing purposes [2]-[4].

In the past, the technical requirements for the provision of these services were hardly attainable by single MGs [5], and thus they were typically supplied by large-scale power generation plants only. Nonetheless, with a recent regulation [6], the European Commission envisioned a novel market framework, endorsing the provision of ancillary services by microgrids' Aggregators (MG-AG), i.e. pools of MGs coordinated by an Aggregator Supervisor (AG), see Figure 1.

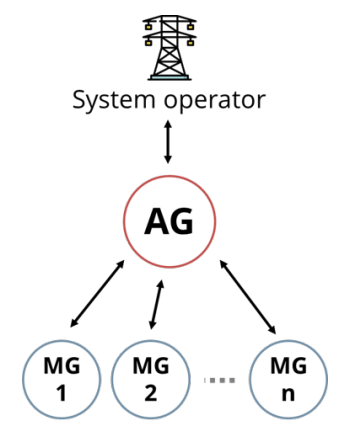

Fig. 1. Schematic of a microgrid aggregator.

Although the aggregation of MGs allows them to meet the minimum technical requirements for the provision of ancillary services [7], it poses significant technological challenges. First, it is necessary to coordinate these MGs to maximize the overall profits, while providing the minimum required amounts of power reserve for the ancillary services provision. Secondly, a privacy-friendly coordination strategy is desirable, meaning that single MGs should not be required to disclose unnecessary internal information. For these motivations, in [8] a distributed day-ahead scheduling approach for MG-AG, guaranteeing the optimal energy management and power reserve provision, has been proposed.

The aim of this paper is to demonstrate the real-world applicability of the algorithm proposed by in [8], verifying its scalability and performances through a real test-bench, with industrial-grade MG simulators and EMS provided by ABB Power Grids, and with real power profiles extracted from the NREL Wind Integration Database and Solar Integration databases [9].

The paper is structured as follows. The optimization approach to schedule the AG operations is briefly described in Section II, since more details are available in [8]. Section III describes the ABB Power Grids e-mesh ${ }^{\mathrm{TM}}$ EMS, while the test bechmark and results are presented in Section IV. Finally, conclusions are discussed in Section V. 


\section{MULTI-STEP SCHEDULING OF MICROGRIDS' AGGREGATORS}

The objective of this section is to give a general overview of the algorithm proposed in [8] and highlight its peculiarities. Every day, within the closure of the Day-Ahead energy market, the AG is required to submit its overall power profile to the System Operators and its availability in terms of capacity reserve. Therefore, the AG must determine the optimal power program of each MG, and the corresponding schedules of internal dispatchable units, such as generators and energy storages, considering:

- The forecasts of non-dispatchable elements, such as RESs and loads;

- The forecasts of the energy prices;

- The minimum amount of power reserve that must be globally provided by the MG-AG for frequency regulation.

Another important service to address, which becomes necessary in presence of many MGs connected to the same distribution network, concerns the regulation of line power flows to avoid over-voltage and over-current events, possibly leading to line congestion issues. The whole optimization procedure presented in [8] is structured in three steps, which are described in the following. The flowchart of the steps' sequence is depicted in Fig. 2.

\section{A. Step 1: Distributed economic dispatch and reserve provi- sion}

Step 1 consists in a preliminary scheduling of MGs' internal units so as to attain the economical optimum, disregarding network constraints, which are not known at this stage. Therefore, just the active power produced or absorbed from each unit is considered. It is thus possible to execute this step solving a multi-temporal optimization problem. A centralized formulation of the problem is certainly straightforward, but shows significant issues hindering its implementation:

- privacy concerns, since a centralized approach would require a full knowledge of the forecasts, the capabilities and the cost functions of MGs' internal units;

- scalability concerns, since a large number of MGs may imply a large and computationally intractable optimization problem;

- applicability concerns, since MGs would be required to give full control to the AG, regardless of the presence of their internal controllers.

On the other hand, a pure decentralized framework for scheduling the MG-AG operations cannot be pursued since MGs must jointly respect the constraints on the minimum amount of power reserve.

In light of these considerations, a distributed optimization problem based on Alternating Direction Method of Multipliers (ADMM) [10] has been adopted. In brief, this method consists in an iterative procedure where each MG locally solves an optimization problem scheduling its units based on some internal prices, which are leveraged by the AG to steer MGs

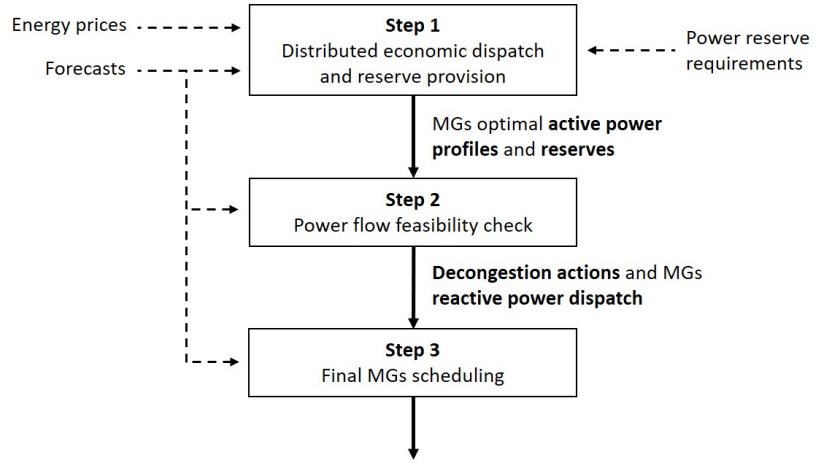

Fig. 2. Flowchart of the microgrids' aggregator scheduling process

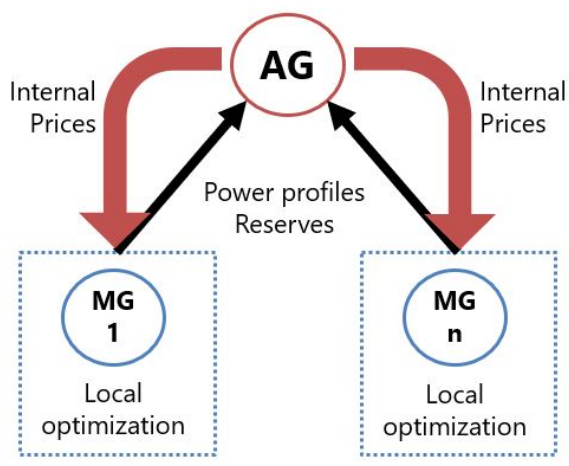

Fig. 3. Flowchart of the microgrids' aggregator scheduling process

towards the global optimum and the satisfaction on the power reserve requirements. The AG uses an internal price for the MGs output power, which can be intended as a cost or a gain depending if the output power is absorbed or delivered. Then, two different prices are used by the AG to incentive MGs to provide upward and downward power reserves, globally satisfying the minimum power requirements. Precisely, the iterative procedure is structured as follows:

1) The AG sends to the MGs the internal prices;

2) The MGs solve in parallel their local optimization problems based on the current prices;

3) The AG gathers the optimal MGs' output power and reserves;

4) The AG updates the internal prices based on the received information in order to steer MGs to the global optimum and satisfy the reserve requirements;

5) Go to 1).

This approach does not require MGs to disclose internal information. Furthermore, it addresses the scalability concerns, as the potentially large centralized problem is partitioned in a large number of smaller sub-problems, which are then solved in parallel by the MGs. Eventually, it does not prescribe a specific formulation for MGs EMS, just requiring some mild assumptions on the underlying optimization problems, see [8]. A schematic, showing the effective interactions between the MGs and the AG, is depicted in Figure 3. 


\section{B. Step 2: Power flow feasibility and reactive power planning.}

Step 2 is necessary to ensure that the active power flows computed in Step 1 are consistent with the constraints on nodal voltages and line currents. In addition, it allows to regulate the reactive power flows inside the distribution network where MGs are connected, minimizing the power losses and ensuring the network electrical feasibility. This is possible since MGs can have a significant role as reactive power producers/consumers, being clusters of several inverter-interfaced generation sources.

Threrefore, it is assumed that, after that Step 1 is executed, MGs communicate their equivalent reactive power capability to the AG. Using this information, each MG can be modelled as an equivalent generator with a defined active power profile (computed in Step 1), together with the communicated active and reactive power capabilities. At this stage, an optimal power flow is carried out considering the distribution network where all MGs are connected. Just in case of feasibility issues, during this optimization step the AG can also require MGs to modify their optimal active power profiles, without however violating the requirements on the minimum active power reserve to provide. This situation occurs just in extreme situations, since the output power of each MG must respect some bounds, dictated for instance by an electric contract with the SO.

At the end of this procedure, the MGs' optimal reactive power profiles are computed, together with the necessary variations on the active power profiles computed by Step 1 .

\section{Step 3: Final scheduling of $M G$ generation units.}

Once Step 1 and Step 2 are executed, the optimal day-ahead active and reactive power profiles are defined for each MG. Therefore, during the last optimization step, MGs schedule the active and reactive power profiles of its generation units considering the requirements from Phase 1 and Phase 2. This step can be carried out in a complete decentralized phase, since the overall power reserve is already guaranteed to respect the minimum bounds.

\section{ABB POWER GRIDS E-MESH ${ }^{\mathrm{TM}}$ EMS DESCRIPTION}

Before discussing the testing results of the described approach, the ABB EMS is presented.

e-mesh ${ }^{\mathrm{TM}}$ EMS is a scalable and highly flexible ABB Power Grids plug-and-play energy management system. The application addresses modern power system challenges by improving energy efficiency, reducing $\mathrm{CO}_{2}$ gas emissions and lowering energy cost at industrial and commercial sites. It is specially designed to manage distributed energy and renewable resources, conventional power generation, and controllable loads like battery energy storage systems and electric vehicle chargers. e-mesh ${ }^{\mathrm{TM}}$ EMS supports scalability options to manage both single site and pools of decentralized energy resources aggregated into virtual power plants. The optimization-based software defines optimal active power set points for every energy resource by evaluating future renewable productions, analyzing load and energy price forecast and asset availability.

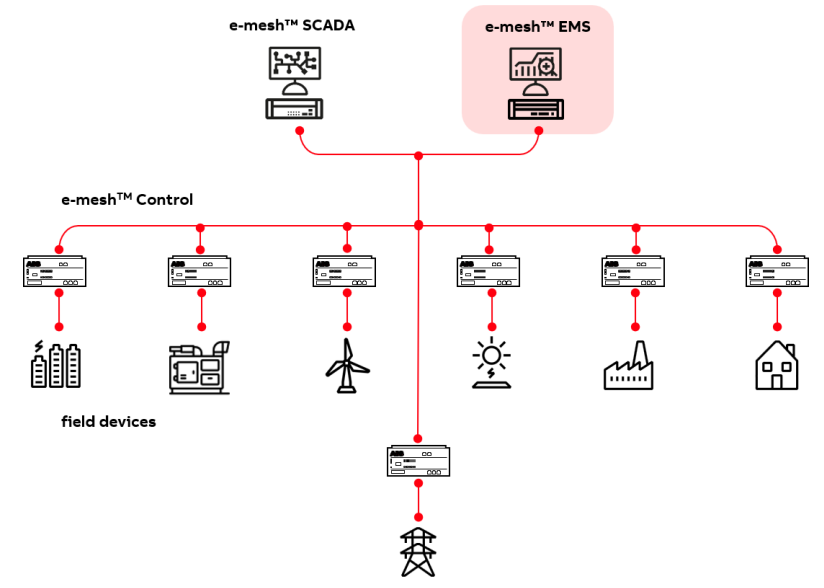

Fig. 4. Typical architecture for ABB Power Grids EMS integrated into the e-mesh ${ }^{\mathrm{TM}}$ stack.

The application is cyber secure and comprises three key modules: Optimize (core module), Connect and Report.

\section{A. EMS Optimize}

The core module uses advanced mathematical models and algorithms to search for optimal power dispatch for all controlled distributed energy resources while taking into account a variety of technical constraints and exploiting information about load, renewable generation and energy price forecast. Most of the implemented constraints depend on the underlying electrical infrastructure and include power exchange limitation with main gird, generation power ramp bounds, storage dynamics and energy balance equations. Furthermore, EMS optimize allows multiple scenarios optimizations with flexible boundary conditions according to user-defined objectives and two different planning horizons:

- day-ahead optimization;

- intra-day optimization.

The day-ahead framework offers the possibility to request a single optimization shot covering 24 hours starting from midnight. Results consist in power programs and capacity reserves to be offered in day-ahead and ancillary service market. It also provide insightful information about future asset set-points. On the other hand, the intra-day optimization is automatically executed by default every 15 minutes with a predefined optimization horizon for real-time energy management applications. Both optimization schemes are formulated as MILP problem, implemented in AMPL algebraic modeling system [11]. The optimization software is generally deployed on an industrial machine with Intel Core i7-6820EQ CPU and 8 GB RAM, which represent the minimum HW requirements.

\section{B. EMS Connect}

ABB Power Grids e-mesh ${ }^{\mathrm{TM}}$ EMS is built on a state-ofthe-art architecture platform, allowing full interoperability and scalability to run at the network edge. Fig. 4 shows how EMS optimize module can be fully integrated with the emesh $^{\mathrm{TM}}$ ecosystem [12]. The product comes with connectivity 
options to integrate SCADA and other similar systems. Customer third-party systems such as forecast providers and trade systems can also be easily connected to the EMS optimize to send or acquire meaningful information. Communication with field controllers is based on MODBUS TCP/IP protocol whereas communication with the SCADA plant is based on Web Rest APIs. Third-party applications are strictly correlated with the customers' requirements and need a customization step instead.

\section{EMS Report}

The reporting module provides a powerful and useful web user interface to monitor optimizations, configure simulations, manage the application life-cycle, configure grid topology and update asset parameters. All acquired information, optimization results and economical KPIs are stored in a local database and leverage intuitive web pages to make them available to any kind of interested user. Moreover, the module is able to generate value-added reports such as revenues from selling excess energy to the grid, costs incurred by purchasing energy from the grid, conventional generators production and $\mathrm{CO}_{2}$ emissions.

\section{TEST SETUP AND RESULTS}

The described day-ahead scheduling algorithm has been tested on an aggregation of 4 different MGs (equipped with dispatchable and non-dispatchable generators, batteries, and loads), which are considered to be connected to the standard IEEE 37-bus system, as depicted in Figure 6. Each MG is equipped with dispatchable generators, storage systems, renewable energy sources and loads. The network data are reported in [13], while the MGs' units characteristics and the energy prices trends are reported in [8]. Considering the ancillary service provision, it is required that at least $1 \mathrm{MW}$ of active power reserve must be available at each time instant. Three MGs, i.e. MG1, MG3, MG4, have been executed in a local machine, while MG2 has been implemented using the ABB e-mesh ${ }^{\mathrm{TM}}$ EMS. Considering the local MGs, their corresponding models and optimization problems, described in details in [8], have been implemented in MATLAB R2019b using CPLEX as a solver. The communication between the $\mathrm{ABB}$ machine and the local machine has been implemented by means of JSON file messages. A schematic of the testing environment is depicted in Figure 5.

The following figures report the outcomes of the described test environment. Concerning the Step 1 of the proposed approach, the distributed algorithm is scalable and computationally efficient, and converged to the optimal value in about 10 iterations, with a total computational time of 20 seconds, considering also the communication time for reading/writing the JSON file. In Figure 7, the overall power program of the MG-AG is reported, while the upwards active power reserve is depicted in Figures 8.

It is worth noting that the $A G$ is able to maintain the aggregated power reserves above the minimum value of $1 \mathrm{MW}$. Moreover, thanks to the multi-step procedure for the day-ahead

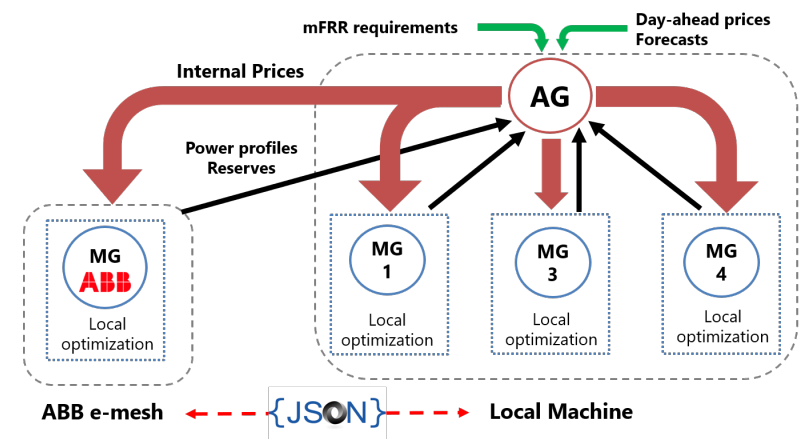

Fig. 5. Schematic of a microgrid aggregator.

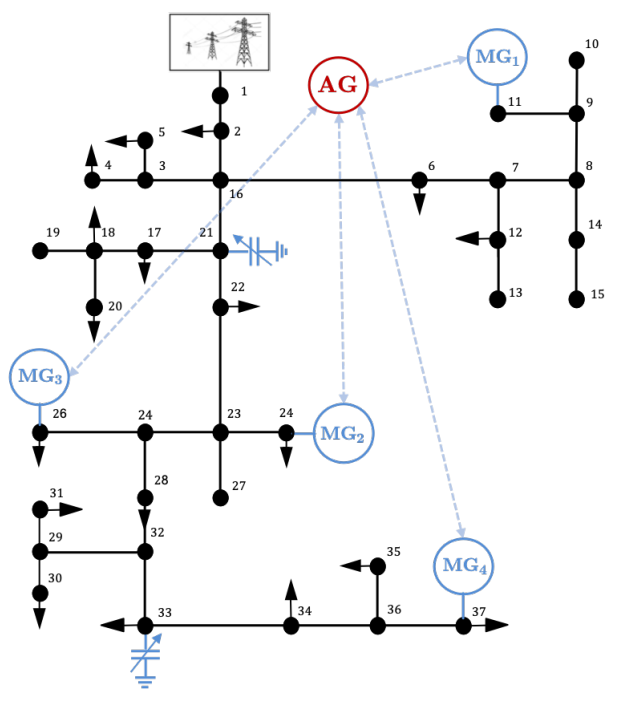

Fig. 6. Schematic of a microgrid aggregator.

scheduling, which considers both the economic optimality and the feasibility of the power flow solution, the scheduled power profiles respect both the line currents and the nodal voltages limitations as shown in Figure 9 and 10. The fulfillment of voltages' and currents' constraints is ensured not only by the control of reactive power flows, but also by slight adjustments of MGs' power profiles, see Figure 11. This results in a variation of the aggregator power program around 17:00.

\section{CONCLUSIONS}

This paper showed the applicability of the Day-ahead Scheduling algorithm proposed in [8], considering an industrial framework with the ABB e-mesh ${ }^{\mathrm{TM}}$ EMS. The testing results show the flexibility and effectiveness of the algorithm to manage microgrids' aggregators, even though different MGs are used and their operations are computed using different machines. Future work may involve the implementation of the algorithm in dedicated electronic dashboards in order to test the method on a real portion of an electric distribution grid to provide ancillary services. 


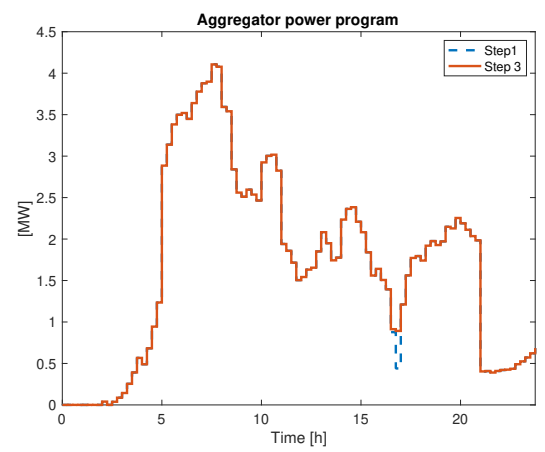

Fig. 7. Power program of the aggregator.

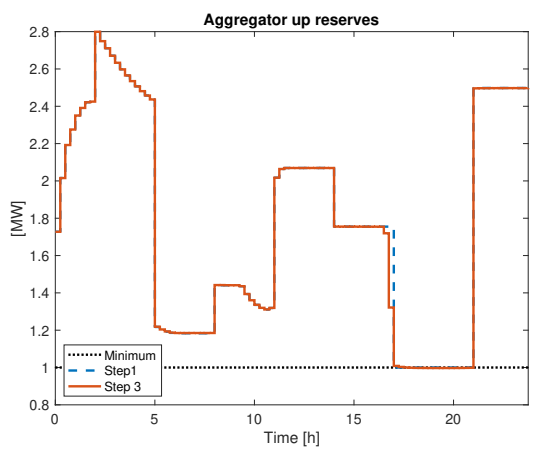

Fig. 8. Programmed upward power reserves of the aggregator.

\section{REFERENCES}

[1] A. Parisio, E. Rikos, and L. Glielmo, "A model predictive control approach to microgrid operation optimization," IEEE Transactions on Control Systems Technology, vol. 22, no. 5, pp. 1813-1827, 2014.

[2] T. Samad, E. Koch, and P. Stluka, "Automated demand response for smart buildings and microgrids: The state of the practice and research challenges," Proceedings of the IEEE, vol. 104, no. 4, pp. 726-744, 2016.

[3] G. Joos, B. Ooi, D. McGillis, F. Galiana, and R. Marceau, "The potential of distributed generation to provide ancillary services," in 2000 Power Engineering Society Summer Meeting (Cat. No. OOCH37134), vol. 3. IEEE, 2000, pp. 1762-1767.

[4] A. La Bella, M. Farina, C. Sandroni, and R. Scattolini, "Microgrids aggregation management providing ancillary services," in Control Conference (ECC), 2018 European. IEEE, 2018, pp. 1136-1141.

[5] C. Yuen, A. Oudalov, and A. Timbus, "The provision of frequency control reserves from multiple microgrids," IEEE Transactions on Industrial Electronics, vol. 58, no. 1, pp. 173-183, 2011.

[6] European Commission, "Commission Regulation (EU) 2017/2195 of 23 November 2017 establishing a guideline on electricity balancing," 2017.

[7] K. Poplavskaya and L. de Vries, "Distributed energy resources and the organized balancing market: A symbiosis yet? case of three european balancing markets," Energy Policy, vol. 126, pp. 264-276, 2019.

[8] A. La Bella, M. Farina, C. Sandroni, and R. Scattolini, "Design of aggregators for the day-ahead management of microgrids providing active and reactive power services," IEEE Transactions on Control Systems Technology, pp. 1-9, 2019.

[9] C. Draxl, A. Clifton, B.-M. Hodge, and J. McCaa, "The wind integration national dataset (wind) toolkit," Applied Energy, vol. 151, pp. 355-366, 2015.

[10] S. Boyd, N. Parikh, E. Chu, B. Peleato, J. Eckstein et al., "Distributed optimization and statistical learning via the alternating direction method of multipliers," Foundations and Trends® in Machine Learning, vol. 3, no. 1, pp. 1-122, 2011.

[11] R. Fourer, D. M. Gay, and B. W. Kernighan, "Ampl. a modeling language for mathematical programming," 1993.

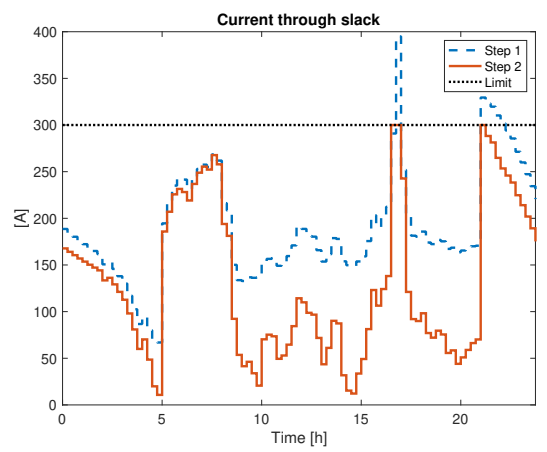

Fig. 9. Current absorbed from the slack node, after Step 1 (dashed line) and after Step 2 (continuous line).

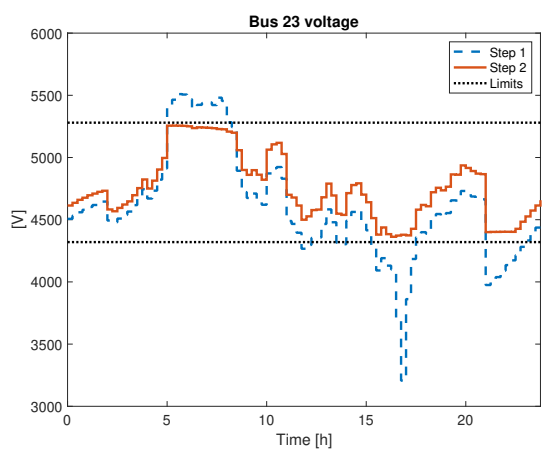

Fig. 10. Voltage profile at bus 23, after Step 1 (dashed line) and after Step 2 (continuous line)
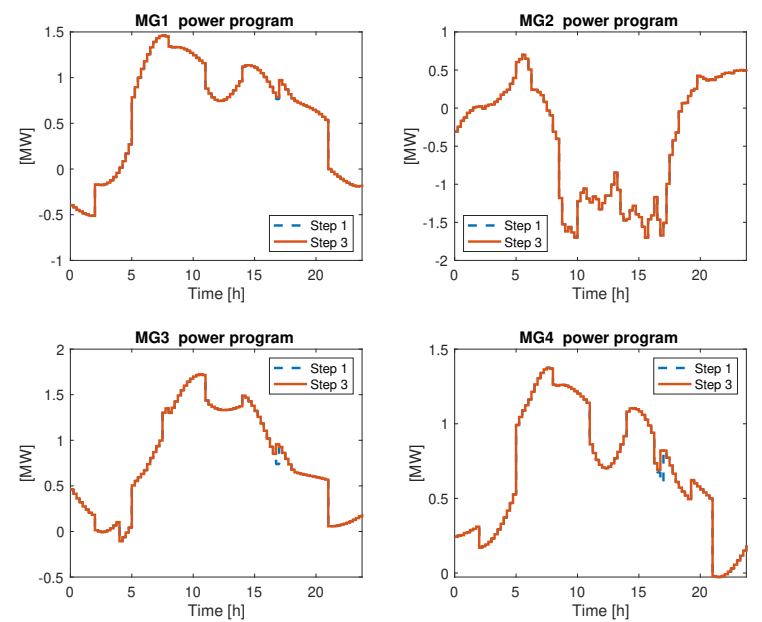

Fig. 11. Power programs of the MGs.

[12] ABB, “ABB Ability e-mesh,” https://new.abb.com/distributed-energymicrogrids/our-offering/e-mesh.

[13] W. H. Kersting, "Radial distribution test feeders," in Power Engineering Society Winter Meeting, 2001. IEEE, vol. 2. IEEE, 2001, pp. 908-912. 